\title{
Optimal Shape Design of Dielectric Micro Lens Using FDTD and Topology Optimization
}

\author{
Young-Seek Chung* and Byungje Lee \\ Department of Wireless Communication Engineering, Kwangwoon University, Seoul 139-701, Korea \\ Sungchul Kim \\ Department of Communication Engineering, Myongji University, Yong-in 449-728, Korea
}

(Received April 3, 2009 : revised April 28, 2009 : accepted April 28, 2009)

\begin{abstract}
In this paper, we present an optimal shape design method for a dielectric microlens which is used to focus an incoming infrared plane wave in wideband, by exploiting the finite difference time domain (FDTD) technique and the topology optimization technique. Topology optimization is a scheme to search an optimal shape by adjusting the material properties, which are design variables, within the design space. And by introducing the adjoint variable method, we can effectively calculate a derivative of the objective function with respect to the design variable. To verify the proposed method, a shape design problem of a dielectric microlens is tested when illuminated by a transverse electric (TE)-polarized infrared plane wave. In this problem, the design variable is the dielectric constant within the design space of a dielectric microlens. The design objective is to maximally focus the incoming magnetic field at a specific point in wideband.
\end{abstract}

Keywords: Optimal shape design, FDTD, Topology optimization, Dielectric microlens

OCIS codes : (220.3620) Lens Design; (230.0230) Optical Devices

\section{INTRODUCTION}

The conventional shape design method is to get better performances by changing the shape of the design space and using the information of the derivative of the objective function with respect to the design variables. It is called Design Sensitivity Analysis (DSA), and it has been established and developed in structural engineering since the 70s [1]. The objective function is chosen to represent the desired performance of the target. In the shape design method, the position or parametric shape vectors are taken as the design variables which specify or control the interface surface between regions.

The authors have proposed the optimal shape design algorithm based on FDTD and DSA [2-4]. However, the previous works had two drawbacks. First, the design sensitivity or the gradient information was not directly evaluated because the adjoint variable equation formulated by FDTD could not be derived in a straightforward way. In order to solve this problem, we employed an adjoint

*Corresponding author: yschung@kw.ac.kr variable equation that was derived from the finite element time domain (FETD) formulation. This adjoint variable equation was then transformed to the coupled Maxwellian differential equations, which were solved by the general Yee's algorithm. Second, in the previous algorithm, since the target shape varies at each design step, a complex mesh generator is needed at each step to generate new meshes. Because of the general Yee's algorithm and the quadrilateral meshes, the design algorithm becomes complex and has many limitations.

Recently, a new design method called Topology Optimization was proposed in the field of structural engineering by Bensoe et al [5]-[9]. They used a large number of adjacent cells in which the material properties such as dielectric constant are allowed to vary continuously between solid and void. By determining that each cell is solid or void so that the design criteria are satisfied, one can obtain an optimally designed shape. This method does not require an initial shape of the design space. Regardless of an initial shape, this technique generates the optimal shape satisfying the design objective. There- 
fore, it is possible to obtain an arbitrary topology of the design space.

In the mid-90's, Dyck introduced the Optimal Material Distribution (OMD) method to the optimal design of magnetic systems [10]. And also, H.B. Lee used this method to obtain a matched load in a waveguide structure at a specific frequency [11].

In this paper, we propose a topology optimization method by using the FDTD method which does not require a complex mesh generator any more. Also, based on the general form of FDTD, the adjoint variable equation can be derived directly and the design sensitivity can be calculated easily.

In order to verify the proposed algorithm, we apply it to the design of a dielectric microlens which is used to detect an infrared plane wave [12]. The design objective is to maximize the magnetic field at a specific point in which a Quantum Well Infrared Photodetector (QWIP) is located [12]. We assumed that the initial design space was uniformly filled with solid dielectric material. The permittivity density of each cell is taken as a design variable. If there is no constraint on the design variable, the design space may be likely to have an unrealizable structure. Thus, in this paper, we imposed a constraint imitating an etching process on the design variables. The results of microlenses designed by the proposed algorithm are successfully compared with those of analytically designed model.

In order to illuminate a plane wave, we adopted the total-field/scattered-field (TF/SF) scheme and we modified it in order to apply it to an infinite substrate. In order to truncate the computational domain, the perfectly matched layer (PML) technique is adopted [15].

\section{TOPOLOGY OPTIMIZATION}

In the topology optimization, the desired space is divided into small cells and the material composition of each cell is taken as a design variable. Since the number of design variables is equal to that of cells in the design space, the number of design variables is larger than for the case of the conventional shape design method. But the larger number of design variables gives more freedom to obtain the shape design solution. By controlling the material composition of each cell, the optimized shape can be constructed approximately. The key concept of this method is how to treat the material composition in order to estimate the objective function, and thus to obtain the final shape of the device. There are two methods to treat the material composition, the homogenization method [5]-[7] and the density method [8]. The homogenization method provides a solid material, physical and mathematical basis for the calculation of the material properties of the composite, or intermediate materials. In order to handle an intermediate material,

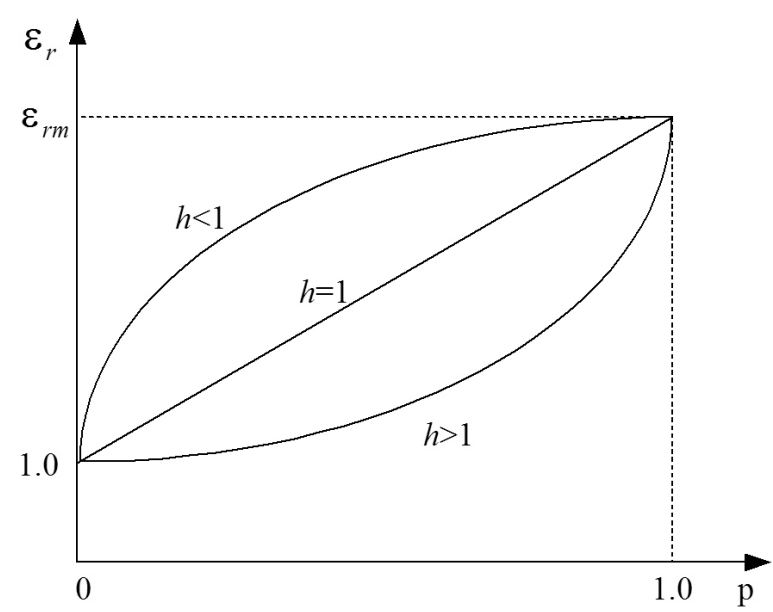

FIG. 1. Relationship between the normalized density $p$ and the relative permittivity $\varepsilon_{r}$. Usually, the value of $h$ is chosen between 2 and 4 considering the convergence rate.

the homogenization method uses the cell with a microhole or an anisotropic material. Thus, the homogenization method must consider the microstructure of the cell, which requires additional solving time. On the other hand, te density method takes the material density of each cell as the design variable and it does not concern the complex microstructure but its characteristics only. That is, the density method regards the complex-structured cells which have the same averaged material density as the same. Thus, the density method is widely used since it is easy to implement and has fewer design variables than the homogenization method. Therefore, in this paper, the density method is adopted.

To apply the density method to the optimization scheme, $p$, the normalized density of the material, is introduced. It takes values between 0 and 1 . Using the normalized density variable $p$, one can represent a relative permittivity as

$\mathcal{E}_{r}=1+\left(\mathcal{E}_{r m}-1\right) p^{h}$

where $\mathcal{E}_{r m}$ is the maximum relative permittivity, and $h$ is the exponent which defines the relationship between the material property and the normalized density. When $p$ is 0 in a specific cell, it means that the cell is empty. And when $p$ is 1 , it means that the cell is entirely filled with dielectric material with $\mathcal{E}_{r}=\mathcal{E}_{r m}$. The values of $p$ between 0 and 1 correspond to intermediate densities which are to be obtained by adjusting $h$. The relation of $\mathcal{E}_{r}$ and $p$ is shown in Fig. 1. When $h$ is large, the initial rate of convergence is fast but the later rate of convergence poor. When $h$ is small, however, the rate of convergence is just the opposite of the previous case. Therefore, considering this trade-off, the value of $h$ is set between 2 and 4 [9]. 


\section{DESIGN SENSITIVITY ANALYSIS}

Using the central difference algorithm, a finite difference form of the time-domain Maxwell equations can be represented as

$$
\begin{aligned}
& \frac{d}{d t}\left(\begin{array}{c}
\varepsilon E \\
\mu H
\end{array}\right)=\left[\begin{array}{cc}
\Sigma_{E} & L_{E} \\
L_{H} & \Sigma_{H}
\end{array}\right]\left(\begin{array}{c}
E \\
H
\end{array}\right)+\left(\begin{array}{c}
J \\
M
\end{array}\right), \text { subject to } \\
& E(t=0)=0, H(t=0)=0
\end{aligned}
$$

where $E$ and $H$ are electric and magnetic field vectors, respectively. $J$ and $M$ are the electric and magnetic current densities, respectively. And the diagonal matrix terms are $\Sigma_{E}=\sigma$ and $\Sigma_{E}=\sigma^{*}$, where $\sigma$ and $\sigma^{*}$ mean the electric and magnetic conductivity, respectively. And the off-diagonal matrix terms, $L_{E}$ and $L_{H}$ are related to the finite difference coefficients for the curl operators. If the time derivatives of (2) are replaced with the central difference relations, (2) becomes the conventional Yee's equation.

In the topology optimization, material properties such as permittivity and permeability can be design variables. In this paper, since the target lens is made of dielectric material, only permittivity becomes the design variable.

To evaluate the optimization performance in wideband, we introduce an objective function

$$
F(p)=\int_{0}^{T_{f}} G(E(p, t), H(p, t)) d t .
$$

where $T_{f}$ is a fixed final time duration and $G$ an arbitrary differentiable function of the transient electromagnetic fields $E, H$, and the design variable vector $p$. Since the change of the design variable $p$ raises the change of the model shape, the transient electromagnetic fields $\mathrm{E}$ and $H$ are dependent on the design variable vector $p$.

Design sensitivity is the gradient of the objective function with respect to design variable vector $p$, which indicates that how the variation of design variables will affect the performance or the objective function. Using the gradient information, one can find the maximum or minimum of the objective function effectively.

The total derivative of $F$ with respect to $p$ is represented as

$$
\frac{d F}{d p}=\int_{0}^{T_{f}}\left(\frac{\partial G}{\partial E} \frac{\partial E}{\partial p}+\frac{\partial G}{\partial H} \frac{\partial H}{\partial p}\right) d t=\int_{0}^{T_{f}}\left(\begin{array}{c}
G_{E} \\
G_{H}
\end{array}\right)^{T}\left(\begin{array}{c}
E_{P} \\
H_{p}
\end{array}\right) d t
$$

where $G_{E}=\frac{\partial G}{\partial E}, G_{H}=\frac{\partial G}{\partial E}, E_{p}=\frac{\partial E}{\partial P}$ and $H_{p}=\frac{\partial H}{\partial P}$. In (4), since $E_{p}$ and $H_{p}$ are implicit functions, we should evaluate $E_{p}$ and $H_{p}$ in an indirect way.

Differentiating (2) with respect to p, we obtain the following equation related to $E_{p}$ and $H_{p}$ as

$$
\frac{d}{d t}\left(\begin{array}{c}
\varepsilon E_{p} \\
\mu H_{p}
\end{array}\right)=\left[\begin{array}{cc}
\Sigma_{E} & L_{E} \\
L_{H} & \Sigma_{H}
\end{array}\right]\left(\begin{array}{c}
E_{p} \\
H_{p}
\end{array}\right)+\left(\begin{array}{c}
J_{p} \\
0
\end{array}\right)
$$

where

$$
J_{P} \equiv \frac{\partial \epsilon}{\partial p} \frac{d \tilde{E}}{d t}
$$

The notation $\sim$ denotes that the variable is held constant for the derivative process with respect to design variable vector $p$. In (5), in order to evaluate $E_{p}$ and $H_{p}$, one should solve (5) for every component of $p$, which means that the number of equations to be solved are equal to the number of components of $p$.

In this work, we introduce the adjoint variable method [5] to reduce the computational burden to solve (5).

Multiplying (5) by the transpose of an adjoint variable vector $(\lambda(\mathrm{t}), \gamma(\mathrm{t}))$, and integrating over the time interval $\left[0, T_{f}\right]$, we have

$$
\int_{0}^{T_{f}}\left(\begin{array}{l}
\lambda \\
\gamma
\end{array}\right)^{T}\left\{\frac{d}{d t}\left(\begin{array}{c}
\varepsilon E_{p} \\
\mu H_{p}
\end{array}\right)-\left[\begin{array}{cc}
\Sigma_{E} & L_{E} \\
L_{H} & \Sigma_{H}
\end{array}\right]\left(\begin{array}{c}
E_{p} \\
H_{p}
\end{array}\right)-\left(\begin{array}{c}
J_{p} \\
0
\end{array}\right)\right\} d t=0 .
$$

Applying the integration by parts to (7) and using $E_{p}(t=0)=0$ and $H_{p}(t=0)=0$, then we have

$$
\begin{aligned}
& \left.\left(\begin{array}{c}
\varepsilon \lambda \\
\mu \gamma
\end{array}\right)^{T}\left(\begin{array}{c}
E_{p} \\
H_{p}
\end{array}\right)\right|_{t=T_{f}}-\int_{0}^{T_{f}}\left\{\frac{d}{d t}\left(\begin{array}{c}
\varepsilon \lambda \\
\mu \gamma
\end{array}\right)^{T}+\left(\begin{array}{c}
\lambda \\
\gamma
\end{array}\right)^{T}\left[\begin{array}{cc}
\Sigma_{E} & L_{E} \\
L_{H} & \Sigma_{H}
\end{array}\right]\right\} \\
& \left(\begin{array}{c}
E_{p} \\
H_{p}
\end{array}\right) d t-\int_{0}^{T_{f}}\left(\begin{array}{c}
\lambda \\
\gamma
\end{array}\right)^{T}\left(\begin{array}{c}
J_{p} \\
0
\end{array}\right) d t=0
\end{aligned}
$$

Since $(\lambda(t), \gamma(t))$ is an arbitrary vector, we can introduce the terminal condition $\lambda\left(T_{f}\right)=\gamma\left(T_{f}\right)=0$ to eliminate the first term of (8), then we have

$$
-\int_{0}^{T_{f}}\left(\begin{array}{l}
\lambda \\
\gamma
\end{array}\right)^{T}\left(\begin{array}{c}
J_{p} \\
0
\end{array}\right) d t=\int_{0}^{T_{f}}\left\{\frac{d}{d t}\left(\begin{array}{c}
\varepsilon \lambda \\
\mu \gamma
\end{array}\right)^{T}+\left(\begin{array}{c}
\lambda \\
\gamma
\end{array}\right)^{T}\left[\begin{array}{cc}
\Sigma_{E} & L_{E} \\
L_{H} & \Sigma_{H}
\end{array}\right]\right\}\left(\begin{array}{c}
E_{p} \\
H_{p}
\end{array}\right) d t
$$

From (4) and (9), we obtain the adjoint variable equation

$$
\begin{aligned}
& \frac{d}{d t}\left(\begin{array}{c}
\varepsilon \lambda \\
\mu \gamma
\end{array}\right)=-\left[\begin{array}{cc}
\Sigma_{E} & L_{E} \\
L_{H} & \Sigma_{H}
\end{array}\right]^{T}\left(\begin{array}{l}
\lambda \\
\gamma
\end{array}\right)+\left(\begin{array}{c}
G_{E} \\
G_{H}
\end{array}\right), \text { subject to } \\
& \lambda\left(T_{f}\right)=\gamma\left(T_{f}\right)=0 .
\end{aligned}
$$

The above equation is a terminal value problem. To handle this easily, we introduce the backward time scheme $\tau \equiv T_{f}-t$ to $(10)$. Then, we have 


$$
\frac{d}{d t}\left(\begin{array}{l}
\varepsilon \bar{\lambda}(\tau) \\
\mu \bar{\gamma}(\tau)
\end{array}\right)=\left[\begin{array}{cc}
\Sigma_{E} & L_{E} \\
L_{H} & \Sigma_{H}
\end{array}\right]^{T}\left(\begin{array}{c}
\bar{\lambda}(\tau) \\
\bar{\gamma}(\tau)
\end{array}\right)-\left(\begin{array}{c}
\bar{G}_{E}(\tau) \\
\bar{G}_{H}(\tau)
\end{array}\right)
$$

where $\bar{\lambda}(\tau)=\lambda\left(T_{f}-t\right), \bar{\gamma}(\tau)=\gamma\left(T_{f}-t\right)$ and $d / d t=-d / d \tau$. Note that since (11) is similar to (2), we can solve and obtain $(\bar{\lambda}(\tau), \bar{\gamma}(\tau))$ by using the conventional FDTD algorithm.

Using (1) and (11), we obtain the design sensitivity as

$$
\frac{d F}{d p}=-\int_{0}^{T_{f}}\left(\begin{array}{c}
\bar{\lambda} \\
\bar{\gamma}
\end{array}\right)^{T}\left(\begin{array}{c}
J_{p} \\
0
\end{array}\right) d t=-\int_{0}^{T_{f}} \bar{\lambda} J_{p} d t
$$

From (1), a derivative of $\varepsilon$ with respect to $p$ is given by

$$
\frac{\partial \varepsilon}{\partial p}=h\left(\varepsilon_{r m}-1\right) p^{h-1}
$$

From (12) and (13), the design sensitivity can be rewritten as

$$
\begin{aligned}
\frac{d F}{d p} & =-\int_{0}^{T_{f}} \bar{\lambda}(t) \frac{\partial \varepsilon}{\partial p} \frac{d \tilde{E}(t)}{d t} d t \\
& =-\int_{0}^{T_{f}} \bar{\lambda}(t) h(\varepsilon-1) p^{h-1} \frac{d \tilde{E}(t)}{d t} d t
\end{aligned}
$$

From (14), we can see that the design sensitivity can be evaluated by solving (11) only once regardless of the number of design variables.

Since in most cases the objective function is a nonlinear function of the design variables, an iterative optimization technique is needed. As iterative optimization algorithm, the steepest descent method is used. The iterative updating process for maximizing the objective function is the following

$$
p_{i}^{k+1}=p_{i}^{k}+\frac{F}{\|d F / d[p]\|^{2}} \frac{d F}{d p_{i}}
$$

where $P_{i}^{k}$ is the $i$ th design variable at the $k$ th optimization iteration. This process is iteratively performed until the objective function value is satisfied with a given tolerance.

\section{PLANE WAVE ILLUMINATION}

If the scattered-field region is the free space, the incident field is equal to a pre-defined incident plane wave. When a plane wave source illuminates the infinite inhomogeneous substrate as shown in Fig. 2, however, the incident field is different from the pre-defined incident field. In the upper scattered-field region, the incident field is the sum of the pre-defined incident field and its reflected field. And in the lower scattered-field region, the incident field is the sum of the pre-defined incident field and its transmitted field [16][17]. Therefore, the

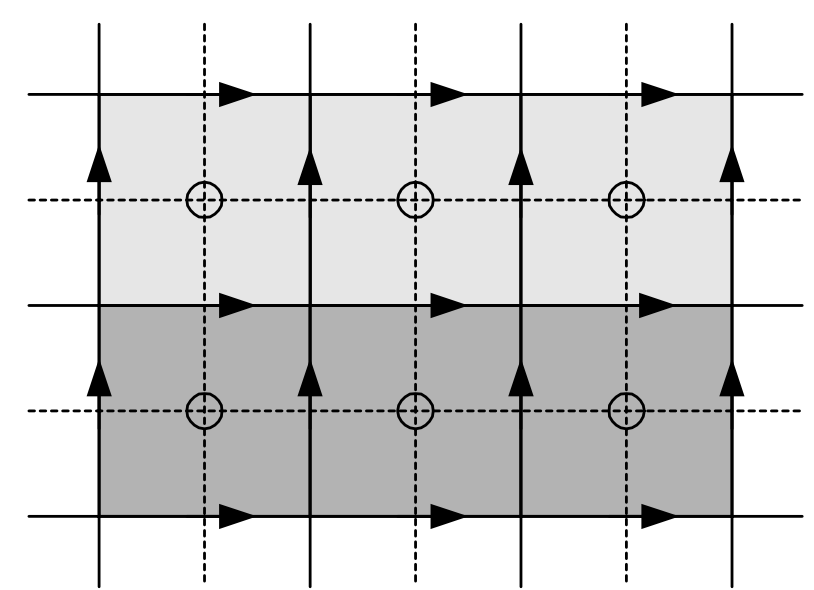

FIG. 2. Assignment of the permittivity in the FDTD cell. The solid circles denote $0 \mathrm{H}_{z}$ fields and the arrow lines denote electric fields $\mathrm{E}_{\mathrm{x}}$ and $\mathrm{E}_{\mathrm{y}}$. The dotted lines construct the FETD cell. The permittivity in the FETD cell may be inhomogeneous. In this case, the averaged value of the permittivities is used.

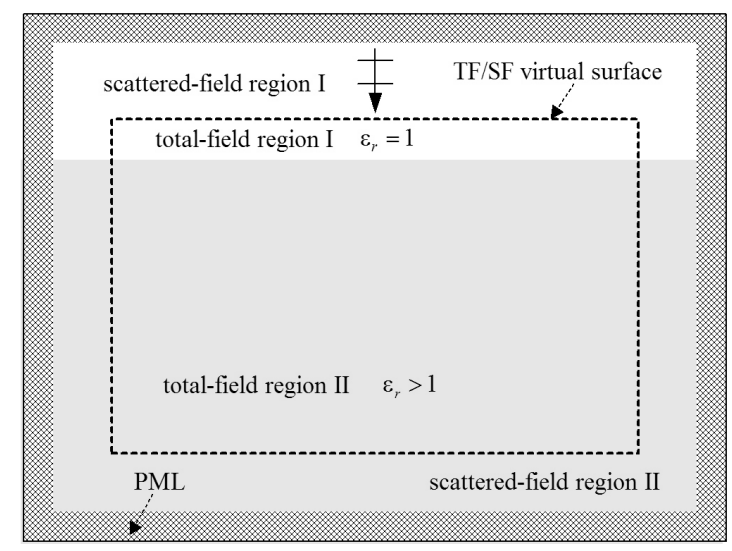

FIG. 3. An infinite inhomogeneous substrate. For illumination of a plane wave, TF/SF virtual surface is located within the computational domain. The computational space is bounded by an absorbing boundary. In this paper, Berenger's PML is used for the absorbing boundary condition.

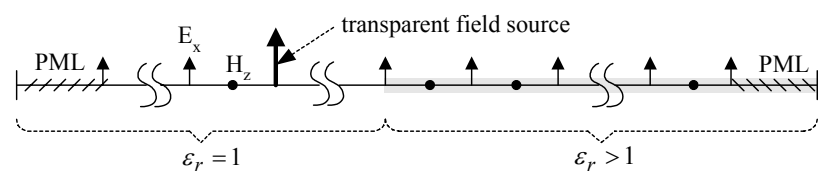

FIG. 4. One dimensional total-field/scattered-field zoning of the FDTD cell. Left and right boundaries are backed by the PML. The inhomogeneous material is located on the right side grayed cells. To implement a hard field source, we adopted the transparent source technique [18]. By using [18], the reflected wave on the material interface passes through the hard source point without any non-physical reflection. 


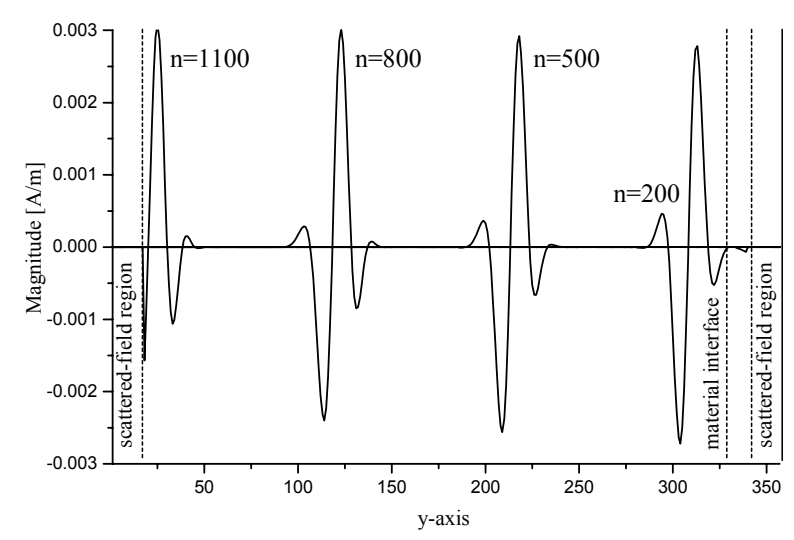

FIG. 5. Magnitude of the magnetic field according to the time iteration. The rightmost waveform is distorted by the reflection on the material interface. One can see that the scattered field in the scattered-field regions is negligible.

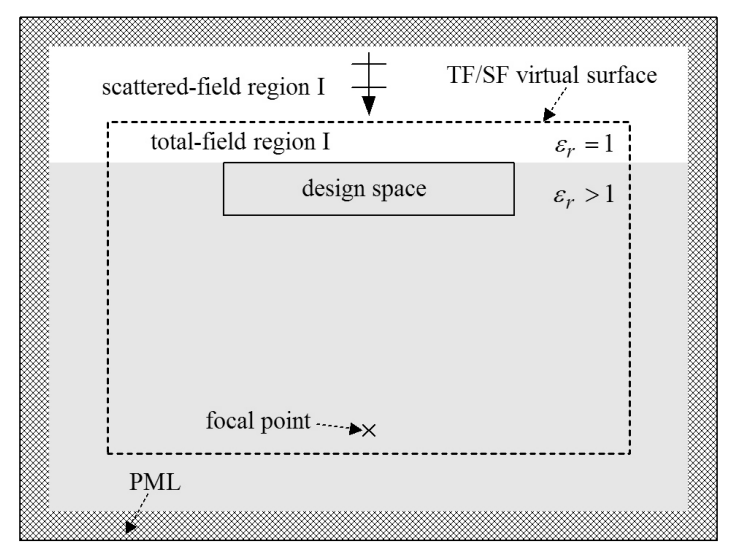

FIG. 6. Initial microlens shape with solid rectangular design space. Since the problem has a symmetric structure, it is sufficient to solve half of it. The size of the cell is $0.35 \times 0.35 \mu \mathrm{m}$. The total number of cells is 510 by 387 . The focal point locates $90 \mu \mathrm{m}$ below the material interface. The initial design is filled with a solid dielectric material. A $\mathrm{TE}^{\mathrm{Z}}$-polarized plane wave is propagating in the direction $\phi=-90^{\circ}$. The scattered fields are absorbed by the PML.

reflected and transmitted fields of the pre-defined incident field must be known a priori at all instants of time. In this paper, in order to implement such a plane wave source, we solve the one-dimensional model that has the same time step as the original analysis model as shown in Fig. 4. Since this one-dimensional model is inhomogeneous, the electromagnetic field reflection occurs and the reflected fields must be absorbed to avoid any non-physical phenomena at the left boundary. Also, the transmitted fields must be absorbed at the right boundary. In order to absorb such reflected and transmitted fields, the one-dimensional PML is adopted. In order to implement a hard type source without any nonphysical retro-reflections, we introduced the transparent field source [18]. Fig. 5 shows the H-field throughout the two-dimensional domain at four different time instants when a $T^{z}$-polarized plane wave is illuminated in the normal direction. Note that the magnetic field in the scattered-field regions is nearly zero.

\section{NUMERICAL EXAMPLE}

The analysis model is shown in Fig. 6 [12], which has a two dimensional shape. This dielectric lens is used for the long-wavelength infrared camera that detects the infrared wave radiated by thermal sources such as intruders. In order to detect the incoming infrared wave even at low power level, an infrared detector should be located at the focal point. Such an infrared camera can find uses in areas such as security, surveillance and earlywarning systems.

The computational domain is divided into cells with dimensions $0.35 \mu \mathrm{m}$ by $0.35 \mu \mathrm{m}$. The total number of cells is 510 by 387 . The focal point is located $90 \mu \mathrm{m}$ below the microlens surface. Considering that the structure is symmetrical, we solved for half the model. The design space is located on the surface and the number of cells is 143 by 43 . At the beginning of the design iteration, we assumed that the design space was filled with the same material as the substrate. The design objective is to obtain the microlens shape that focuses the magnetic field of the incoming plane wave in a wideband so that the infrared detector senses an emitting source well.

The incident wave was assumed to be a pulsed $\mathrm{TE}^{\mathrm{Z}}$ plane wave that was a Gaussian modulated by a sine function with the center frequency of $20 \mathrm{THz}$. We assumed that the plane wave propagates toward the $-\mathrm{y}$-axis. In order to estimate the spectral density of the magnetic field at the focal point, we defined the function $G$ as

$$
G(E(t), H(t)) \equiv \frac{1}{2}\left(\left.H_{z}(t)\right|_{f}\right)^{2}
$$

Thus, the objective function is defined as

$$
F=\frac{1}{2} \int_{0}^{T_{f}}\left(\left.H_{z}(t)\right|_{f}\right)^{2} d t
$$

where $H_{z}(t) \mid \mathrm{f}$ is the magnetic field at the focal point.

If there is no constraint on the design variable, there may be cases in which it is difficult to realize the obtained shape. Therefore, in order to obtain a manufacturable shape, we impose the following constraints that imitate the etching process of semiconductor technology.

(1) The interior cells are excluded from the design variable. Such cells are called inactive cells. That is, only the exterior cells become design variables. Such cells are called active ones.

(2) If the relative permittivity of an active cell becomes 


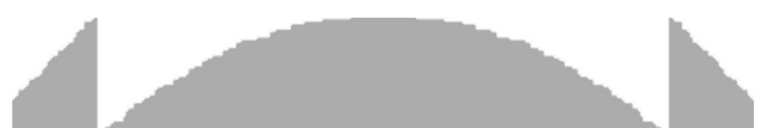

(a)

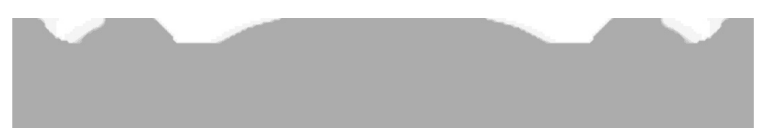

(b)

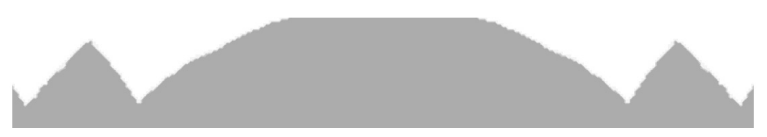

(c)

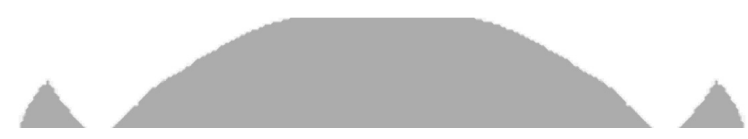

(d)

FIG. 7. Shape of the lens in [12] and shape variation of the design space according to the design iteration when $n=2.0$ : (a) [12] (b) after 10 iterations (c) 50 iterations (d) 85 iterations.

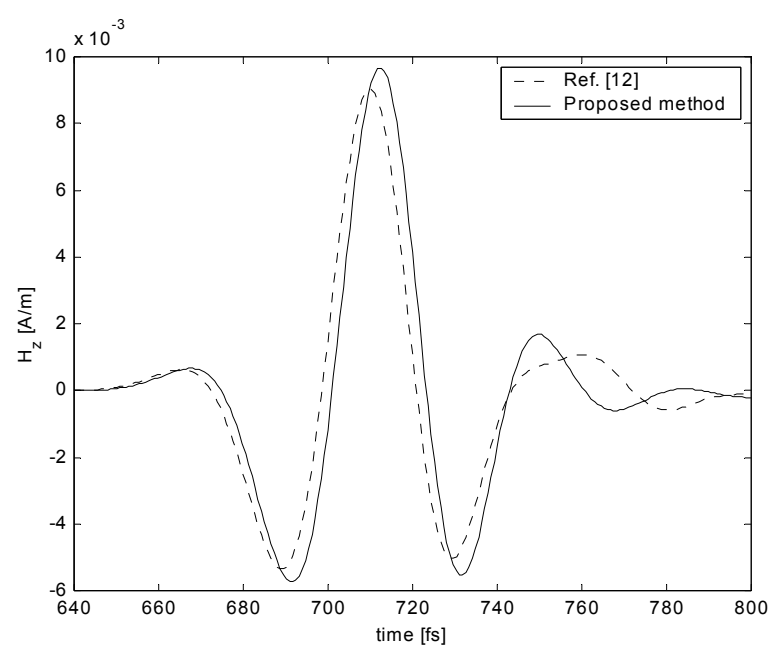

FIG. 8. Time domain magnetic field at the focal point. The dotted line is for [12] and the solid line is for proposed method after 85 iterations.

less than a fixed value in the design process, the neighboring inactive cells are switched to active ones. The direction of the simulated etching process is assumed to be downwards. Therefore, an interior inactive cell becomes an active one just when the above active cell is sufficiently etched.

(3) An almost empty cell that is equivalent to a completely etched cell is changed to an inactive one and excluded from the design variables permanently.

To collimate an incoming plane wave at the focal point, the shape of a dielectric lens which has an index

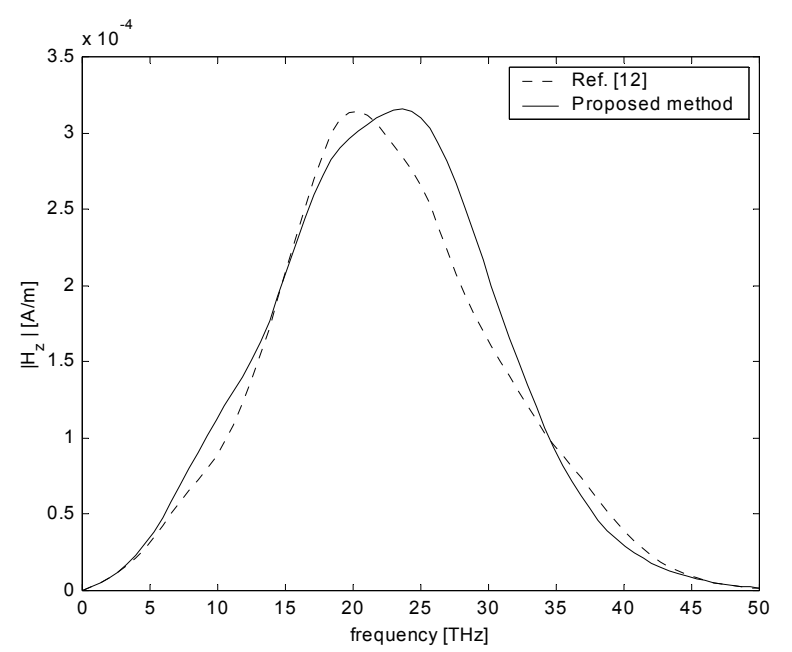

FIG. 9. Fourier transformation of the results of Fig. 8.

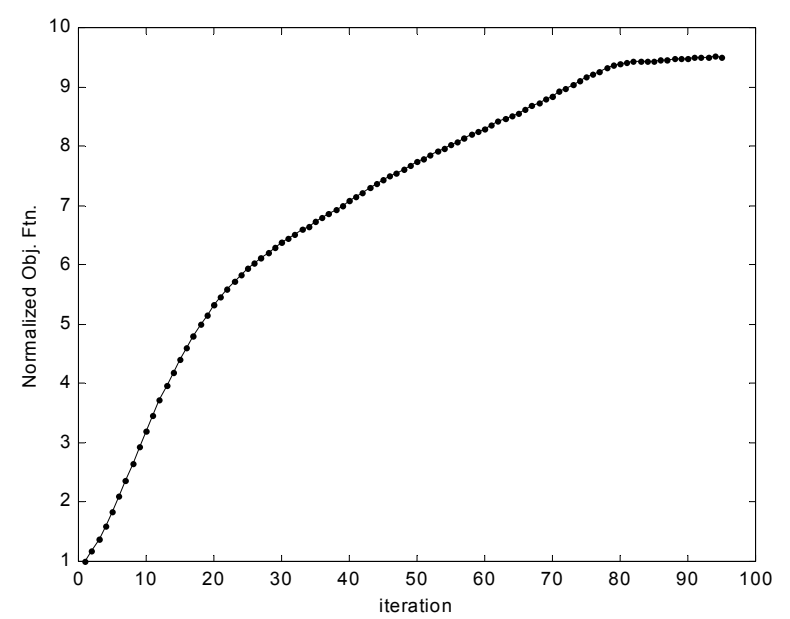

FIG. 10. Convergence characteristic of the objective function $F$. In this figure, the objective function is normalized by the initial value of $F$. It is achieved after 80 iterations.

of refraction with $\mathrm{n}>1$ must be convex. Refractive index is defined as the inverse square root of the relative permittivity. And the size, weight, and power loss by the dielectric lens can be reduced by zoning one of its surfaces [19][20]. Fig. 7(a) shows the lens shape as stated above [12]. Fig. 7(b) (d) shows the shape variation according to design iteration when $n=2$.

One can find that the lens shapes of [12] and our result are similar. But, while the lens shape of [12] is designed to maximally focus magnetic field at $f=20 \mathrm{THz}$ in the frequency domain, the lens shape of Fig. 7(d) is designed to maximally focus the total magnetic field energy of the incoming plane wave over a broad frequency.

Fig. 8 compares the time domain magnetic field at the focal point of [12] and our results after 85 iterations. Fig. 9 shows the Fast Fourier Transformation (FFT) results corresponding to Fig. 8. In this figure, while the maximum intensity of the magnetic field of [12] lies at 


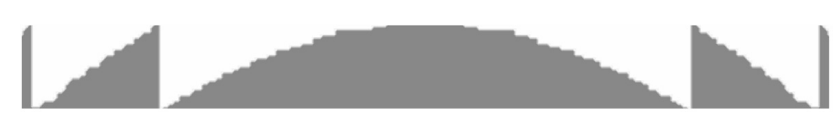

(a)

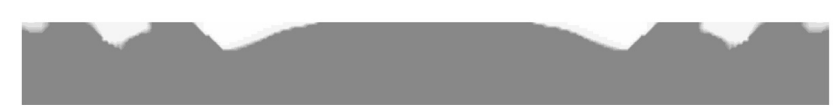

(b)

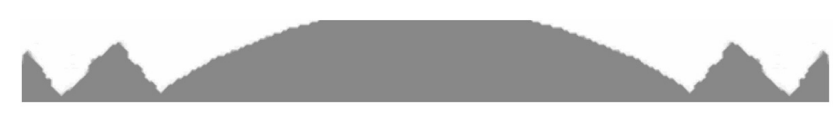

(c)

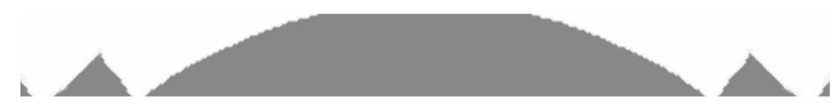

(d)

FIG. 11. Shape of the lens in [12] and shape variation of the design space when $n=2.5$ : (a) [12] (b) after 10 iterations (c) 50 iterations (d) 65 iterations

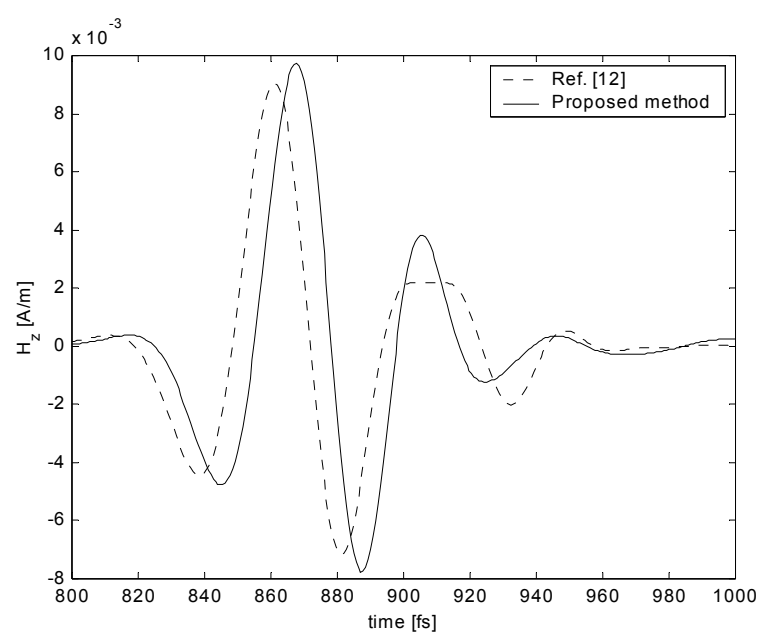

FIG. 12. Time domain magnetic field at the focal point. The dotted line is for [12] and the solid line is for proposed method after 65 iterations.

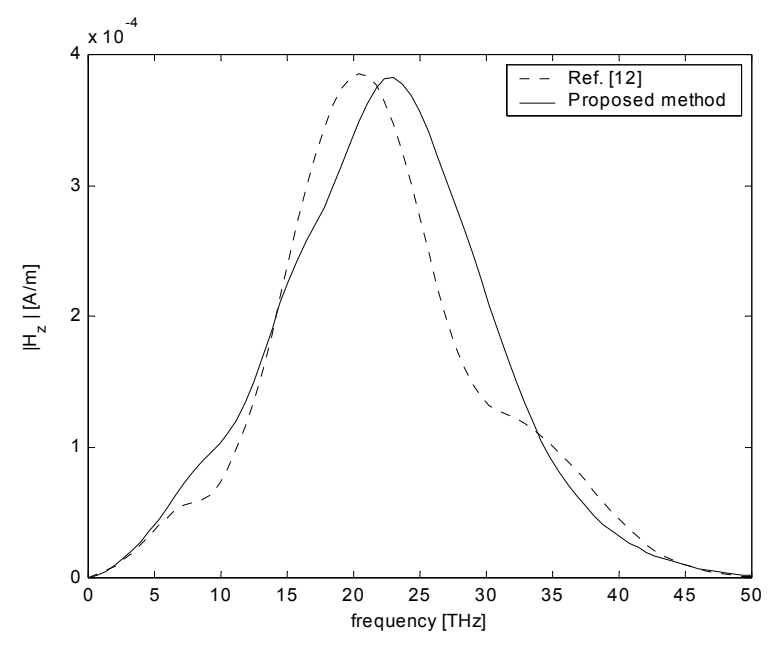

FIG. 13. Fourier transformation results of Fig. 12. $f=20 \mathrm{THz}$ as expected, that of our model lies at about $f=23 \mathrm{THz}$. This is because the incoming plane wave used in this paper is not exactly symmetric with respect to $f=20 \mathrm{THz}$. Fig. 10 shows the convergence characteristic of the objective function. One can see that convergence is obtained after 80 iterations. This is due to the above constraints. Fig. 11 shows the lens shapes when the refractive index is 2.5. Similarly, the lens shape of Fig. 11(a) is designed to maximally focus magnetic field at $20 \mathrm{THz}$. Fig. 12 compares the time domain magnetic fields at the focal point for [12] and our designed lens. Fig. 13 shows the FFT results corresponding to Fig. 12. In this figure, one can see that the designed lens shifts the frequency of the maximum magnetic intensity towards a higher frequency than that of [12].

\section{CONCLUSIONS}

This paper has presented an optimal design technique using the FDTD method and Topology Optimization, which allows the design of a dielectric microlens in a wideband. This dielectric microlens may be installed in the infrared camera used for a surveillance system. In order to operate even at low power level, the incoming plane wave needs to be maximally focused. Thus the design objective is to obtain the shape of microlens to maximally focus the incoming wave at a focal point. Using the topology optimization technique, we can obtain the optimally designed shape of the model without mesh regeneration. However if there is no constraint on the design variables, one may obtain unrealizable structures. Thus, in order to design a feasible structure of the microlens, we introduced the constraints imitating the etching process in semiconductor technology and obtained a reasonable shape of the microlens. The results were compared with that of an analytically designed model.

\section{ACKNOWLEDGMENT}

The present research has been conducted by the Research Grant of Kwangwoon University in 2008.

\section{REFERENCES}

1. E. J. Haug, K. K. Choi, and V. Komkov, Design Sensitivity Analysis of Structural System (Academic Press, New York, USA, 1986).

2. Y. S. Chung, C. Cheon, I. H. Park, and S. Y. Hahn, "Optimal design method for microwave device using FDTD and design sensitivity analysis," IEEE Trans. MTT. 48, 2289-2296 (2000).

3. Y. S. Chung, J. Ryu, C. Cheon, I. H. Park, and S. Y. Hahn, "Optimal design method for microwave device 
using time domain method and design sensitivity analysis : I. FETD case”, IEEE Trans. Magn. 37, 3289-3293 (2001).

4. Y. S. Chung, C. Cheon, I. H. Park, and S. Y. Hahn, "Optimal design method for microwave device using time domain method and design sensitivity analysis : II. FDTD case," IEEE Trans. Magn. 37, 3255-3259 (2001).

5. M. P. Bendsoe and N. Kikuchi, "Generating optimal topologies in structural design using a homogenization method," Computer Methods in Applied Mechanics and Engineering 71, 197-224 (1988).

6. M. P. Bendsoe, "Optimal shape design as a material distribution problem," Structural Optimization 1, 193-202 (1989).

7. K. Suzuki and N. Kikuchi, "A homogenization method for shape and topology optimization," Computer Methods in Applied Mechanics and Engineering 93, 291-318 (1991).

8. H. P. Mlejnek and R. Schirrmacher, "An engineer's approach to optimal material distribution and shape finding," Computer Methods in Applied Mechanics and Engineering 106, 1-26 (1993).

9. R. J. Yang, "Multidiscipline topology optimization," Computer \& Structures 63, 1205-1212 (1997).

10. D. N. Dyck and D. A. Lawther, "Automated design of magnetic devices by optimizing material distribution," IEEE Trans. Magn. 32, 1188-1193 (1996).

11. H. Lee, "Computer aided optimal design methods for waveguide structures," Ph.D. Dissertation, Seoul National
University (1995).

12. D. S. Katz, "FDTD modeling of dielectric lens," http:// pat.jpl.nara.gov/public/dsk/FDTD (1998).

13. J. Choi, J. Jung, S. Park, and T. Kwon, "The compound refractive lens for hard X-ray focusing," J. Opt. Soc. Korea 11, 76-81 (2007).

14. G. Kweon, Y. Choi, and M. Laikin, "Fisheye lens for image processing application,” J. Opt. Soc. Korea 12, 79-87 (2008).

15. A. Taflove, Computational Electrodynamics: The FiniteDifference Time-domain Method (Artech House, Boston, USA, 1995).

16. K. Demarest, R. Plumb, and Z. Huang, "FDTD modeling of scatterers in stratified media," IEEE Trans. Antennas Propag. 43, 1164-1168 (1995).

17. P. B. Wong, G. L. Tyler, J. E. Baron, E. M. Gurrola, and R. A. Simpson, "A three-wave FDTD approach to surface scattering with applications to remote sensing of geophysical surfaces," IEEE Trans. Antennas Propag. 44, 504-514 (1996).

18. J. B. Schneider, C. L. Wagner, and O. M. Ramahi, "Implementation of transparent sources in FDTD simulation," IEEE Trans. Antennas Propag. 46, 1159-1168 (1998).

19. C. A Balanis, Antenna Theory: Analysis and Design (John Wiley \& Sons, Singapore, 1982).

20. H. Jasik, Antenna Engineering Handbook (McGrawHill, New York, USA, 1961). 